\title{
Endovascular Management for Salvage of Failing Arteriovenous Fistula
}

\author{
Atef Abdel Hameed, MD; Mohamed Ismail, MD; Ramez Mounir, MD
}

Department of Vascular Surgery, Ain Shams University, Cairo, Egypt

Background: Failure of arteriovenous access for hemodialysis is a challenging problem. Maintaining the patency of this access is one of the most important corner stones in the care of patients with chronic renal failure. Recently, percutaneous transluminal balloon angioplasty (PTA) is considered the first line of treatment for dealing with this issue.

Aim: The purpose of this retrospective observational study was to evaluate the primary and secondary patency rates after initial endovascular intervention to restore and preserve the patency of failing autogenous arteriovenous fistulae and to identify the potential factors that affect the durability of the procedure.

Methods: The results of 56 patients with failing autogenous arteriovenous fistulae were retrospectively analyzed after being treated with percutaneous transluminal balloon angioplasty from November 2014 to December 2018. Technical \& clinical success rates were reported. The variables, including patients' demographics, co-morbidities, medications, fistula age, fistula type, site, number of lesions and degree of stenosis were analyzed and correlated with primary and secondary patency rates.

Results: The mean age of the fistulae included in the study since their creation was $17.4 \pm 9.6$ months, most of which were radiocephalic fistulas (64.3\%). The most common cause of autogenous access dysfunction was $90-99 \%$ stenosis while the most common site of stenosis was juxta-anastomotic. Technical and clinical success rates of the study population were $92.9 \%$ and $89.3 \%$, respectively. The mean primary and secondary patency were 12.3 and 19.9 months, respectively. Primary patency rates at 6,12,18 and 24 months were $79 \%, 64 \%, 57 \%$ and $44 \%$ respectively. Secondary patency rates at 6,12,18 and 24 months were $85 \%, 76 \%, 68 \%$ and $52 \%$ respectively. Patient age $\geq 60$ years old was associated with reduced post-PTA primary patency $(P<0.001)$ and secondary patency $(P=0.018)$. Dyslipidemia showed marked decrease in both primary and secondary patency rates $(P<0.001)$. Insulin intake $(P<$ 0.001 ) was a predictor of primary patency decrease while use of antiplatlets was to a less extent predictor of secondary patency loss $(P=0.004)$. Radiocephalic fistulae had short primary patency $(P=0.016)$, while stenotic lesions $>90 \%$ showed a significant decrease $(P<0.001)$ in primary patency more obvious than with secondary patency $(P=0.006)$. Lesions at arteriovenous anastomosis were significantly associated with decrease in primary patency $(P<0.001)$. Statins were the only medications associated with longer primary patency $(P<0.001)$. Age of fistula and number of lesions were independent factors.

Conclusion: Endovascular treatment is both safe and effective in managing failing autogenous arteriovenous fistulas. Although its technical and success rates are high, primary and secondary patency rates are still questionable. Dyslipidemia, insulin, statin and antiplatelet intake together with age of patient, degree and site of stenosis are potential risk factors that affect these rates.

Key words: Autogenous arteriovenous fistula, failing fistula, access salvage, hemodialysis.

\section{Introduction}

The vascular access guidelines of the National Kidney Foundation Kidney Disease Outcomes Quality Initiative (NKF-K/DOQUI) stated that autogenous arteriovenous fistula (AVF) is the best available access for providing hemodialysis. ${ }^{1}$ There is a positive correlation between the duration and frequency of hemodialysis and patient morbidity and mortality. ${ }^{2}$ All hemodialysis access are inevitably subjected to fail with time. ${ }^{3}$ Approximately $20-30 \%$ of hospital admissions of hemodialysis patients (average 2 admissions per year per patient) are due to access failure and dysfunction. ${ }^{4}$ Restoration of dysfunctional access could be achieved by either percutaneous balloon angioplasty, surgical procedure or a combination of both methods. ${ }^{5}$ Recently, endovascular management, when possible, is considered the first line to manage access stenosis because of its improved outcomes and the convenience of performing the procedure in the outpatient setting. ${ }^{6}$ The purpose of this retrospective observational study was to evaluate the primary and secondary patency rates after initial endovascular intervention, to restore and preserve the patency of failing autogenous arteriovenous fistulae and to identify the potential factors that affect the durability of the procedure.

\section{Patients and methods}

From November 2014 to December 2018., the medical records of 56 patients with failing autogenous arteriovenous fistula for hemodialysis who underwent 
access salvage by percutaneous balloon angioplasty were retrospectively analyzed. Follow up was done every 6 months for 2 years. The patients included in the study were those who had been referred to our vascular surgery unit for angiographic evaluation due to failure to perform their usual hemodialysis after at least 3 months of usage of autogenous arteriovenous fistula. This was decided when one or more of the following criteria appeared: decrease or absence of thrill, difficulty in cannulation, prolonged bleeding time after dialysis, development of collateral veins or persistent elevation in dynamic venous pressures unexplained by needle position or size. We excluded patients with immature fistulae, thrombosed fistulae, previous surgical or endovascular attempts to salvage the access and patients with accompanied central venous hypertension.

\section{Technique}

All procedures were performed at Ain Shams University hospitals. All patients were provided oral and written consent before angiography. All procedures were performed in hybrid OR theater on outpatient setting.

First, a thorough examination for the AVF was done to determine the most probable cause of access failure.
Cannulation site was selected either via radial artery, brachial artery, cephalic or basilic vein. Puncture was done under local anesthesia using duplex ultrasound. A micropuncture needle (Cook, Bloomington, IN, USA) was used for cannulation of radial artery at the anatomical snuff box. Fistulogram was done for the whole tree till the distal out flow to detect all stenosis sites and degrees together with presence of any accessory veins with significant importance. A 6-F sheath was inserted. A standard guide wire (Terumo, Tokyo, Japan) was then passed across the lesion either antegrade or retrograde according to the site of cannulation. Some lesions were crossed using 0.018 or 0.014 wire with the help of supporting catheter (Trailblazer, Medtronic, Minneapolis, MN, USA). Balloon angioplasty was performed using 5-9 balloons (Admiral Xtreme, Medtronic, Minneapolis, MN, USA) or high-pressure balloons (Mustang, Boston scientific, Natck, MA, USA). If the lesion was tight to be dilated by conventional balloon, a cutting balloon (Boston scientific, Natck, MA, USA) was used. Bare metal stents with $6-8 \mathrm{~mm}$ in diameter were used in case of recoil in native vein lesions after venous anastomosis against sufficient balloon dilatation. On imaging, if accessory vein was identified to be greater than half of the main fistula, we surgically ligated it.

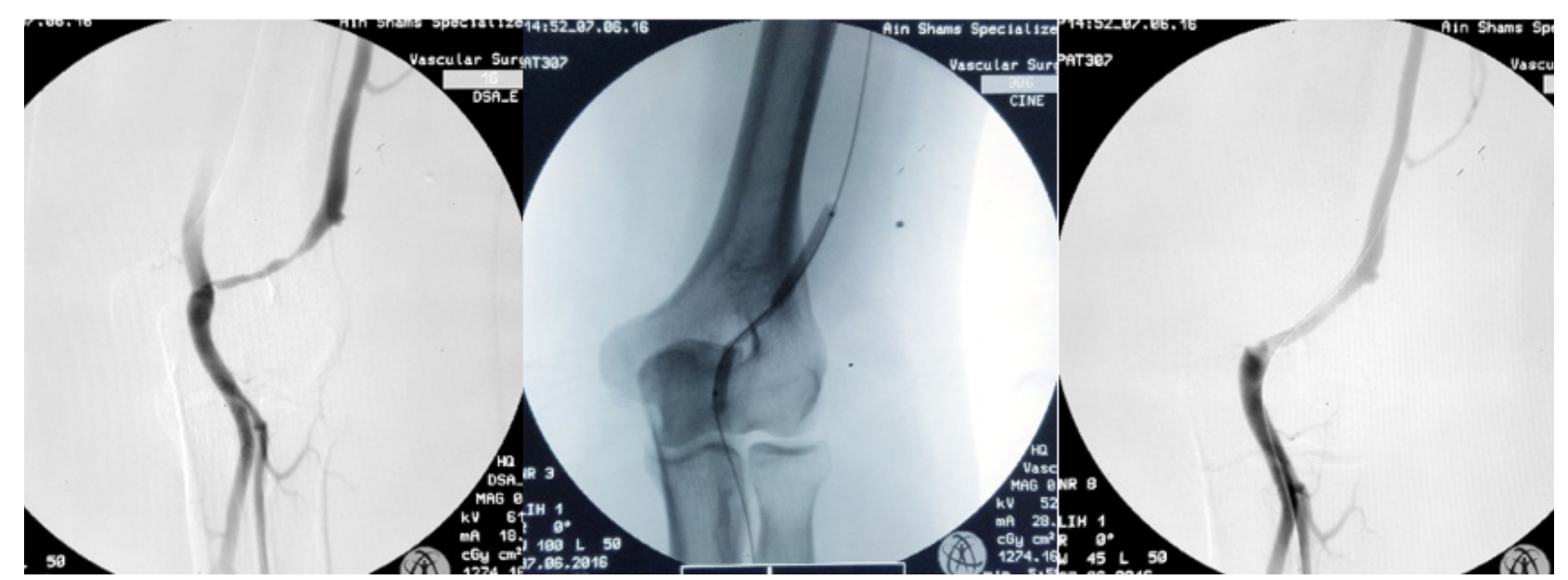

Fig 1: Endovascular salvage of dysfunctional brachiocephalic fistula due to significant stenosis at the juxta-anastomotic vein. 

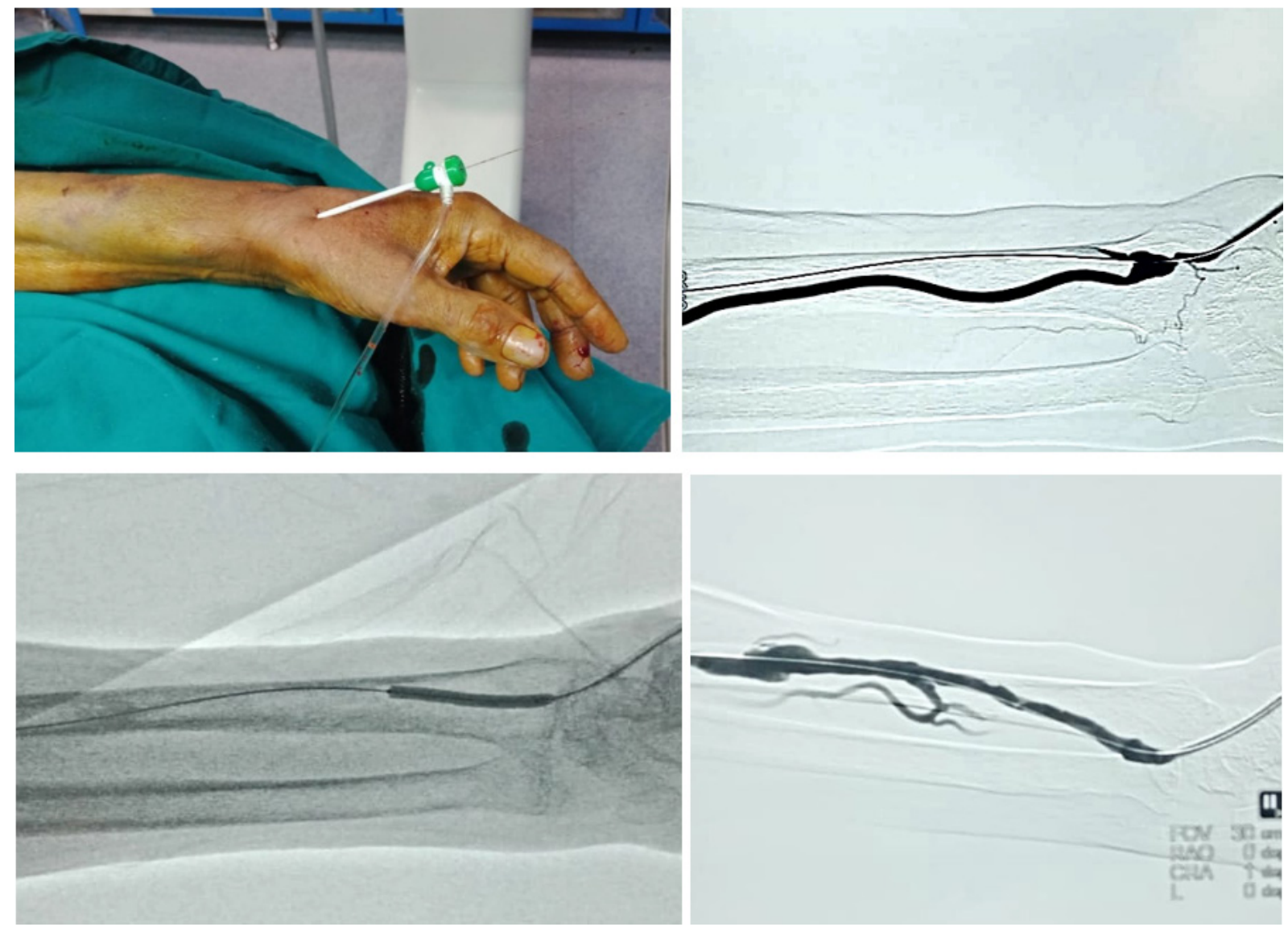

Fig 2: Endovascular salvage of totally occluded radiocephalic fistula. Access via radial artery at the anatomical snuff box.

\section{Definitions and Study Endpoints}

"Technical success" was defined by restoration of flow in vascular access with residual stenosis less than $30 \%$ without major complication that indicate termination of the procedure. "Clinical success" was defined when the patient can perform at least one successful hemodialysis session after the procedure of angioplasty. "AVF age" was the time between fistula creation and the time of our initial PTA. "Venous stenosis" was defined as $50 \%$ or greater decrease in lumen diameter compared to the adjacent segment of the AVF vein with associated hemodynamic or clinical abnormalities. "Degree of stenosis" was defined as the narrowest diameter compared with adjacent vein immediately upstream of the target lesion and was classified into 4 categories; $50-74 \%, 75-90 \%, 90-99 \%$ and $100 \%$ total occlusion.

"Site of stenosis" was measured at the following parts of vascular access:

1. Native artery.

2. Arteriovenous anastomosis.

3. Juxta-anastomoic vein (initial $2 \mathrm{~cm}$ of the fistula).

4. Venous outflow (>2 $\mathrm{cm}$ from anastomosis).

5. Distal outflow (above elbow joint for radiocephalic fistulae and above mid humerus for brachial fistulae).

"Primary patency" was defined as the interval following intervention until the need to repeat PTA, vascular access failure or study end (after 2 years of follow up), whichever occurred first. "Secondary patency" was defined as the interval after intervention till the fistula was surgically ligated, revised or excluded due to inability to be used regardless subsequent trials of PTAs, new access creation, kidney transplantation loss during follow up period or death.

\section{Statistical analysis}

Summaries of the categorical factors were described using frequencies and percentages. The mean and standard deviation were used to summarize continuous measure distributions. Chi-square test was used to compare qualitative variables.

\section{Results}

56 patients with failing autogenous AVF underwent endovascular treatment during the study period. Patency of the access after our initial PTA was followed every 6 months for 2 years. Regarding the demographics and clinical characteristics of the patients (Table 1), we treated $32(57.1 \%)$ males and $24(42.9 \%)$ females with mean age $56 \pm 11$ years. The most common co-morbidities accompanying the patients were hypertension, 33 patients (59\%) and diabetes, 32 patients (57\%). Statins and Insulin 
were the commonest drugs taken by the patients. During follow up 2 (3.6\%) patients underwent kidney transplantation and one patient $(1.8 \%)$ died.

Table 1: Patients demographic and clinical characteristics

\begin{tabular}{lc}
\hline \multicolumn{1}{c}{ Characteristics } & $\mathbf{n = 5 6}$ \\
Age (years) & $56 \pm 11$ \\
Gender n (\%) & \\
- Male & $32(57.1 \%)$ \\
- Female & $24(42.9 \%)$
\end{tabular}

Drug Intake (Medications) ${ }^{a}$

- Antiplatelet

$19(33.9 \%)$

- Statin

$25(44.6 \%)$

- Insulin

$25(44.6 \%)$

- Oral anticoagulant

$4(7.1 \%)$

- Oral hypoglycemic

$7(12.5 \%)$

Co-morbidity n (\%)

- Diabetes

$32(57.1 \%)$

- Hypertension

$33(58.9 \%)$

- Dyslipidemia

$28(50 \%)$

- Coronary artery disease

$10(17.8 \%)$

- Cerebrovascular accident

$8(14.3 \%)$

$19(33.9 \%)$

Smoking $\mathrm{n}(\%)$

$2(3.6 \%)$

- Kidney transplant

$1(1.8 \%)$

- Patient death

a Use of medications prior, during and post PTA.

The mean age of the fistulae included in the study since their creation was $17.4 \pm 9.6$ months, most of them were radiocephalic fistulas in $36(64.3 \%)$ patients, while we operated upon 14 (25\%) brachiocephalic fistulas. Brachiobasilic fistulas showed to be the least to be affected in $6(10.7 \%)$ patients. The majority were in the left side in $34(60.7 \%)$ of cases. A history of previous ipsilateral placement of central venous catheter was noted in 30 (53.6\%) of patients. Characteristics of the fistulae are reported in (Table 2).

Table 2: Arteriovenous fistula characteristics

\begin{tabular}{lc}
\hline Characteristics & $\mathbf{n = 5 6}$ \\
\hline Right side n (\%) & $22(39.3 \%)$ \\
Left side & $34(60.7 \%)$ \\
Age of fistula (months) & $17.4 \pm 9.6$ \\
Type of Fistula n (\%) & \\
• Radiocephalic & $36(64.3 \%)$ \\
$\bullet \quad$ Brachiocephalic & $14(25 \%)$ \\
• Brachiobasilic & $6(10.7 \%)$ \\
$\begin{array}{l}\text { Previous ipsilateral placement of } \\
\text { central venous catheter }\end{array}$ & $30(53.6 \%)$ \\
\hline
\end{tabular}

Our puncture was via radial artery in 35 (62.4\%) procedures, 5 times (9\%) through brachial artery, 13 $(23.2 \%)$ via cephalic vein and 3 times $(5.4 \%)$ via basilic vein.

As for the lesions (Table 3), the most common cause of access dysfunction was $90-99 \%$ stenosis in 21 $(37.5 \%)$ patients while $20(35.7 \%)$ patients had $75-$ $89 \%$ stenosis. To less extent, 12 (21.4\%) patients had $50-74 \%$ stenosis while the cause of failure was due to total occlusion in $3(5.4 \%)$ of patients. The most common site of stenosis was juxta-anastomotic in 22 (39.3\%) of patients followed by arteriovenous anastomosis in 14 (25\%) AVFs, 11 (19.6) in venous outflow, $5(9 \%)$ in distal outflow and $4(7.1 \%)$ native arteries were affected. Most of the lesions were at one site in $40(71.4 \%)$ of cases. Presence of accessory vein was observed in $12(21.4 \%), 5$ of which showed to be significantly large and were surgically ligated using local anesthesia in the same session. 


\begin{tabular}{|c|c|c|}
\hline & Category & n (\%) \\
\hline \multirow[t]{4}{*}{ Degree of stenosis } & Total occlusion $100 \%$ & $3(5.4)$ \\
\hline & Stenosis $90-99 \%$ & $21(37.5)$ \\
\hline & $75-89 \%$ & $20(35.7)$ \\
\hline & - $50-74 \%$ & $12(21.4)$ \\
\hline \multirow[t]{5}{*}{ Site of lesion } & Native artery & $4(7.1)$ \\
\hline & Arteriovenous anastomosis & $14(25)$ \\
\hline & Juxta -anastomosis & $22(39.3)$ \\
\hline & Venous outflow & $11(19.6)$ \\
\hline & Distal outflow & $5(9)$ \\
\hline \multirow[t]{3}{*}{ Number of lesions } & 1 site & $40(71.4)$ \\
\hline & 2 sites & $10(17.9)$ \\
\hline & $\geq 3$ sites & $6(10.7)$ \\
\hline Presence of significant accessory vein & & $12(21.4)$ \\
\hline
\end{tabular}

We used traditional balloons for dilatation of the lesions in all the procedures either primarily or for predilatation. High pressure balloons were required 13 times $(23.2 \%)$ while a need for cutting balloon was in $3(5.4 \%)$ procedures. Self-expandable stents were deployed due to postdilatation recoil twice $(3.6 \%)$ in this study.

The technical and clinical success rates of the study population were $92.9 \%$ (52 of 56 AVFs) and $89.3 \%$ (50 of 56 AVFs), respectively. Technical failure to salvage the access occurred in 4 patients, two of them due to failure to pass the wire and cross the lesion and the other two due to severe recoil after balloon dilatation. Puncture site hematoma occurred in 4 (7.1\%) cases only which was conservatively managed.

The mean primary and secondary patency were 12.3 and 19.9 months, respectively. Primary patency rates at $6,12,18$ and 24 months were $79 \%, 64 \%, 57 \%$ and $44 \%$ respectively. Secondary patency rates at $6,12,18$ and 24 months were $85 \%, 76 \%, 68 \%$ and $52 \%$ respectively.

The variables, including age of the patients above 60 years, gender, co-morbidities, medications, fistula age, type of fistula, number of lesions, site and degree of stenosis were evaluated as potential risk factors for primary and secondary patency rates.

Regarding primary patency, patient age $\geq 60$ years old was associated with reduced post-PTA primary patency $(P<0.001)$, no significant change was noted with gender difference. Dyslipidemia showed marked decrease in primary patency $(P<0.001)$ together with insulin intake $(P<0.001)$. Radiocephalic fistulae had short primary patency $(P=0.016)$, while stenotic lesions > 90\% showed a significant decrease $(\mathrm{P}<$ $0.001)$. Statins were the only medications associated with longer primary patency $(P<0.001)$. Lesions at arteriovenous anastomosis was significantly associated with decrease in primary patency $(P<0.001)$. Age of fistula and number of lesions were independent factors regarding the same rate.

By comparing the same variables to secondary patency rates, we found reduced rate with age $\geq 60$ years $(P=0.018)$, significant reduction with dyslipidemia $(P<0.001)$. Use of antiplatlets was a predictor of secondary patency decrease $(P=0.004)$. Lesion stenosis $>90 \%$ showed short secondary patency $(P=0.006)$ but not as those rates with primary patency.

\section{Discussion}

Vascular access is one of the most valuable factors affecting the quality and longevity of life in patients with end stage renal disease on regular hemodialysis. The durability of this access is still questionable due to suboptimal patency rates. ${ }^{7}$ Primary patency rates for autogenous arteriovenous fistulae were reported to be $72 \%$ at 6 months and only $51 \%$ at 18 months. ${ }^{8}$ Attempt to salvage a failing vascular access for hemodialysis is considered as important as creating it. ${ }^{9}$ Endovascular management with balloon angioplasty for stenosis causing access dysfunction is associated with excellent success rates, low complication rates, and promising long-term patency rates. ${ }^{10}$ However, compared to surgery, it has worse primary patency and nictitates repetition to achieve similar secondary patency levels. ${ }^{11}$

Our technical success rate was $92.9 \%$ while clinical 
success rate was $89.3 \%$. All the procedures were done on outpatient setting, so no hospital stay was required. No major complications or vessel rupture occurred. No postprocedural infection was noted.

Our mean primary and secondary patency were 12.3 and 19.9 months, respectively. Primary patency rates at $6,12,18$ and 24 months were $79 \%, 64 \%, 57 \%$ and $44 \%$ respectively while secondary patency rates at $6,12,18$ and 24 months were $85 \%, 76 \%, 68 \%$ and $52 \%$ respectively. This indicates a distinguished rate of restenosis and the need to deal with the situation by repetition of PTA or surgical intervention.

In our study, attention was given to determine the potential factors that affect the durability of initial PTA for failing autogenous AVF and lead to recurrent dysfunction. Dyslipidemia and old age above 60 years showed to decrease both primary and secondary patency rates. This may be related to atherosclerosis and the process of intimal hyperplasia.

Although diabetes mellitus was not associated with any change in post PTA primary or secondary patency, we found that the group of diabetic patients taking insulin were subjected to decrease in primary patency period more than those on oral hypoglycemic treatment. We relate this mostly to the state of long standing diabetes or poor control with the insulin group.

In our analysis, statins were the only medications associated with longer primary patency. The Study of Heart and Renal Protection (SHARP) randomized trial found a reduction in vascular access occlusive events in patients taking statins and cholesterol inhibitors. ${ }^{12}$

A study of use of clopidogrel in patients with AVF, whose primary outcome measure was fistula failure at 8 weeks, found a significantly lower failure rate in the clopidogrel group compared to placebo (5.26\% compared to $21.6 \%) .{ }^{13}$ We think that the decrease in secondary patency rate with our patients taking antiplatlets was due to the nature of peripheral arterial disease they suffer from rather than the effect of the medication itself.

Tight and anastomotic stenotic lesions showed to be liable to restenosis in the study follow up period, while there was no significant effect of age of fistula or number of lesions in the same access.

The limitations of our study were lack of control group, small number of population, no enough data about the cause of recurrent access failure post PTA. There was selection bias in this cohort as the decision to abandon the fistula and surgically correct it or create a new one versus additional PTA differs according to surgeon preference. Lastly, exclusion of thrombosed access and accompanying central venous stenosis might affect the study results.

\section{Conclusion}

Endovascular treatment is both safe and effective in managing failing autogenous arteriovenous fistulas. Although its technical and success rates are high, primary and secondary patency rates are still questionable. Dyslipidemia, insulin, statin and antiplatlet intake together with age of patient, degree and site of stenosis are potential risk factors that affect these rates.

\section{References}

1. Vascular Access: Clinical practice guidelines of the European society of vascular surgery. Eur $\boldsymbol{J}$ Vasc Endovasc Surg. 2018; 55: 753-754.

2. Santoro D., Benedetto F., Mondello P., et al: Vascular access for hemodialysis: Current perspectives. Int. J Nephrol Dis. 2014; 7:281-294.

3. Suttie SA, Ponnuvelu G, Henderson N, et al: Natural history of upper arteriovenous fistulae for chronic hemodialysis. J Vasc Access. 2012; 13: 332-337.

4. Himmelfarb J: Hemodialysis. $\mathbf{N}$ Engl J Med. 2010; 363: 1833-45.

5. Trosini N, Chisci E, Frosini P, et al: Hybrid simultaneous treatment of thrombosed prosthetic grafts for hemodialysis. J Vasc Access. 2014; 15: 396-400.

6. Thomas M, Nesbitt C, Ghouri M, Hansrani M: Maintenance of hemodialysis vascular access and prevention of access dysfunction: Review. Ann Vasc Surg. 2017; 43: 318-327.

7. Nikam MD, Ritchie J, Jayanti A, et al: Acute arteriovenous access failure: Long-term outcomes of endovascular salvage and assessment of co variates affecting patency. Nephron. 2015; 129:241-6.

8. Huber TS, Carter JW, Carter RL, et al: Patency of autogenous and polytetrafluoroethylene upper extremity arteriovenous hemodialysis accesses: A systematic review. J Vasc Surg. 2003; 38: 10051011.

9. Bloembergen WE, Hakim RM, Stannard DC, et al: Relationship of dialysis membrane and causespecific mortality. Am J Kidney Dis. 1999; 33: 1-10.

10. Nassar GR: Endovascular management of the "failing to mature" arteriovenous fistula. Tech Vasc Interventional Rad. 2008; 11: 175-180.

11. Long B, Brichart N, Lermusiaux $P$, TurmelRodrigues $L$, et al: Management of perianastomotic stenosis of direct wrist autogenous radial-cephalic arteriovenous accesses for dialysis. J Vasc Surg. 2011; 53: 108-114. 
12. Baigent $C$, Landray $M L$, Reith $C$, et al: The effects of lowering LDL cholesterol with simvastatin plus ezetimibe in patients with chronic kidney disease (Study of Heart and Renal Protection): A randomized placebo-controlled trial. Lancet. 2011; 377: 2181-2192.
13. Ghorbani A, Aalamshah $M$, Shahbazian $H$, et al: Randomized controlled trial of clopidogrel to prevent primary arteriovenous fistula failure in hemodialysis patients. Indian J Nephrol. 2009; 19: 57-61. 